\title{
A Proposed Framework for Smart Home Systems Design and Adoption
}

\author{
Oluoha, O. \& Ebem, D.U. \\ aDepartment of Computer Science \\ Faculty of Physical Sciences \\ University of Nigeria \\ Nsukka, Nigeria \\ Email: d_blackdiamond@yahoo.com \\ Phones: +2348181320874, +2348033854983
}

\begin{abstract}
The Smart Home concept is an extensive technological concept which have seen a major resurgence in recent years. They are aimed at automating daily living activities, central monitoring and control of the home, power consumption optimization, greater security, and ensuring a healthier living condition among other things. The capabilities of the Smart Home are further enhanced when combined with the Internet of Things (IOT) and cutting edge technologies such as embedded artificial intelligence. However, the Smart Home design is beset by a number of issues including a lack of universal technical standards and platform fragmentation. This paper is aimed at generating operational guidelines and a design framework for Smart Home architecture. An extensive survey of literature is carried out to investigate the general architecture, existing models and designs of the Smart Home System in other to establish how current models impact on the operations of the smart home. We then highlight the major challenges to the smart home architecture and why these exist. Finally, we attempt to discuss what possible solutions can mitigate identified challenges. A general framework is then proposed for the design of smart homes, which helps overcome identified drawbacks to the Smart Home architecture.
\end{abstract}

Keywords: Smart Home, Automation, Internet of Things (IOT), Home Area Network (HAN).

\section{CISDI Journal Reference Format}

Oluoha, O. \& Ebem, D.U. (2019): A Proposed Framework for Smart Home Systems Design and Adoption. Computing, Information Systems, Development Informatics \& Allied Research Journal. Vol 9 No 1.Pp 15-28. Available online at www.cisdijournal.org DOI Affixed - https://doi.org/10.22624/AIMS/CISDI/V10N1P3.

\section{BACKGROUND TO THE STUDY}

It is difficult to reach a consensus as to the definition of a Smart Home, as most definitions could get very vague especially when it comes to distinguishing between building intelligence and building automation (Eugeny, 2015). Some scholars go as far as to assert that many social systems can be smart without necessarily basing their activities on Information and Communication Technologies (Jucevičius, Patašienè \& Patašius, 2014). This difficulty as to arriving at a consensus for the definition of a Smart Home could be due to the very fast pace of technological changes in this area. However, De Silva, Morikawa and Petra I.M. (2012) and Zhang, Shah and Papageorgiou (2013) both describe a Smart Home as a home-like environment that has ambient intelligence, automatic control, keeps track of and respond to its inhabitants behaviours. The main aim of every Smart Home System design is to attain to desired home automation levels, ensure adequate security, conserve energy consumption in the building and ensure the wellbeing of its inhabitants. FIG. 1 below presents a general architecture of the smart home system. 


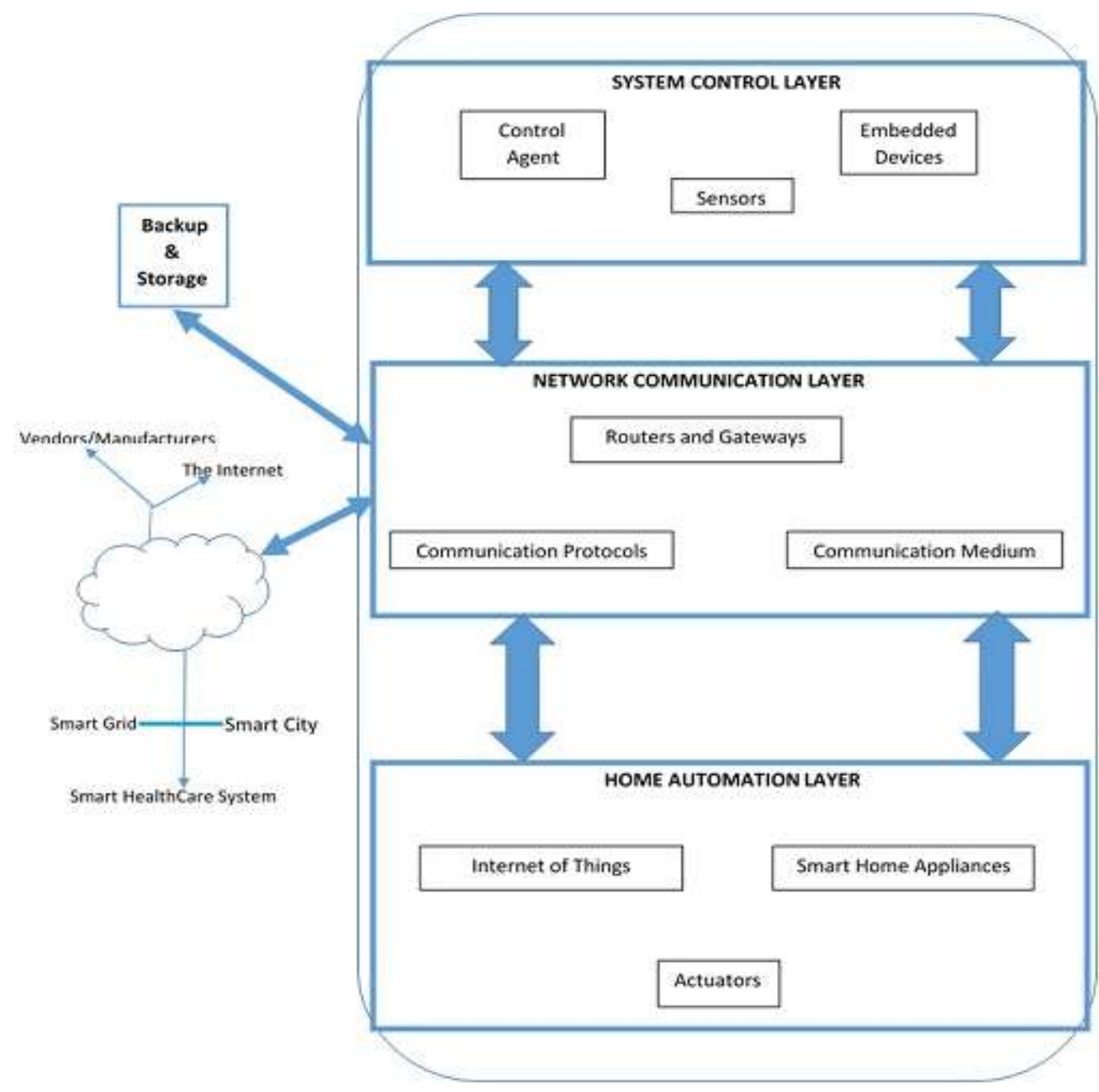

Fig 1: General Architecture of a Smart Home System

The ever increasing number of connected device in today's homes and buildings presents a world of innumerable opportunities for future homes. The Internet of Things (IOT) has been touted as the next revolutionary technology in transforming the Internet into a fully integrated environment. Praveen and Bagavathi (2016) assert that the Internet of Things (IoT) has necessitated the concept of Smart Homes and cities. Automated monitoring and response is an integral aspect of smart home systems and the implementation of smart infrastructure approaches have been shown to be quite appropriate for achieving sustainable development in developing countries. It greatly enhances infrastructure development and environmental conditions in such target environments in other to achieve sustainable urban development and expansion (Soyinka, Siua, Lawansonb \& Adeniji, 2016). Smart homes hold great prospects in the areas of energy efficient buildings, building safety, aiding people with disabilities (such as the physically and mentally challenged), more secure buildings, the list is seemingly endless. The ever evolving world of "smart things" has also brought about warble devices, which could be integrated into the Smart Home fabric, presenting an unending horizon of possibilities. 
The loT combined with wireless sensing technologies has extraordinary potentials, and will fast become the focus for cutting edge remote monitoring and control systems due to their distributed intelligence and pervasive connectivity (Ghayvat, Mukhopadhyay, Gui \& Suryadevara, 2015). Innovation has been a hallmark of the human thought pattern throughout history, and this has played out strongly in the field of Smart Home design and development.

\subsection{Statement of Problem}

The ever increasing number of connected device in today's homes and buildings presents a world of innumerable advantages and opportunities for future homes. However, Smart buildings differ greatly from normal building both in their design and operation. Implementing Smart Home designs therefore come with several unique hurdles. First, Smart Home systems exhibit a very high level of heterogeneity both in hardware setup and user needs. Second, there is no universally accepted standard for Smart Home design. This implies that interconnecting and interoperating devices in the Smart Homes (in terms of shared resources and information) can be a major nightmare.

\subsection{Objective}

The main objective of this study is to critically look at the general architecture of the smart home system and its functional parts, analyse technologies and platforms used, pinpoint major flaws in current smart home architectural frameworks and present possible solutions in proposed framework.

\section{METHODOLOGY}

\subsection{The Research Design}

An extensive literature survey was carried out to study the very complex interrelationships between housing, technology, daily life and working activities. In other to understand the challenges of the smart home design, it is imperative to understand the functional design and sub-systems in the Smart Home architecture and their complex interaction so as to comprehend which communication protocols to implement in other to ensure overall Smart Home system performance (Roberto, Giuseppe, Pilloni \& Luigi, 2014). In today's Smart Home systems, implantables (such as a pacemaker, etc), wearables (such as T-shirts, wrist straps and similar wearable devices) and microsystems (fall detectors, ECG, etc) have been incorporated into the overall design of Smart Homes. Smart Homes contain different kinds of sensors and actuators, working on diverse platforms and protocols, geared towards achieving varied objectives. By understanding the strengths and weaknesses of the technologies and protocols, one can better understand underlying challenges and harness their communications and collaboration capabilities towards building a Smart Home architecture that meets all set expectations.

\subsection{Smart Home Architecture}

In this study, the Smart Home was subdivided into four main functional parts or sub-systems. These include; Home Security and Surveillance Sub-system, Home Energy Management Sub-system, Home Entertainment and Multimedia Sub-system and Home HealthCare Sub-system (see FIG. 2). Depending on its architecture and main aim, a Smart Home could be designed to place more emphasis on one or more of these sub-systems or could be designed to equally integrate the capabilities of all four sub-systems into the Smart Home. For instance, a Smart Home could be designed for the aged or handicapped, with greater focus on the Home Health Care Sub-system. It should also be noted that the different functional parts of the sub-systems demand different resource requirements and challenges. 


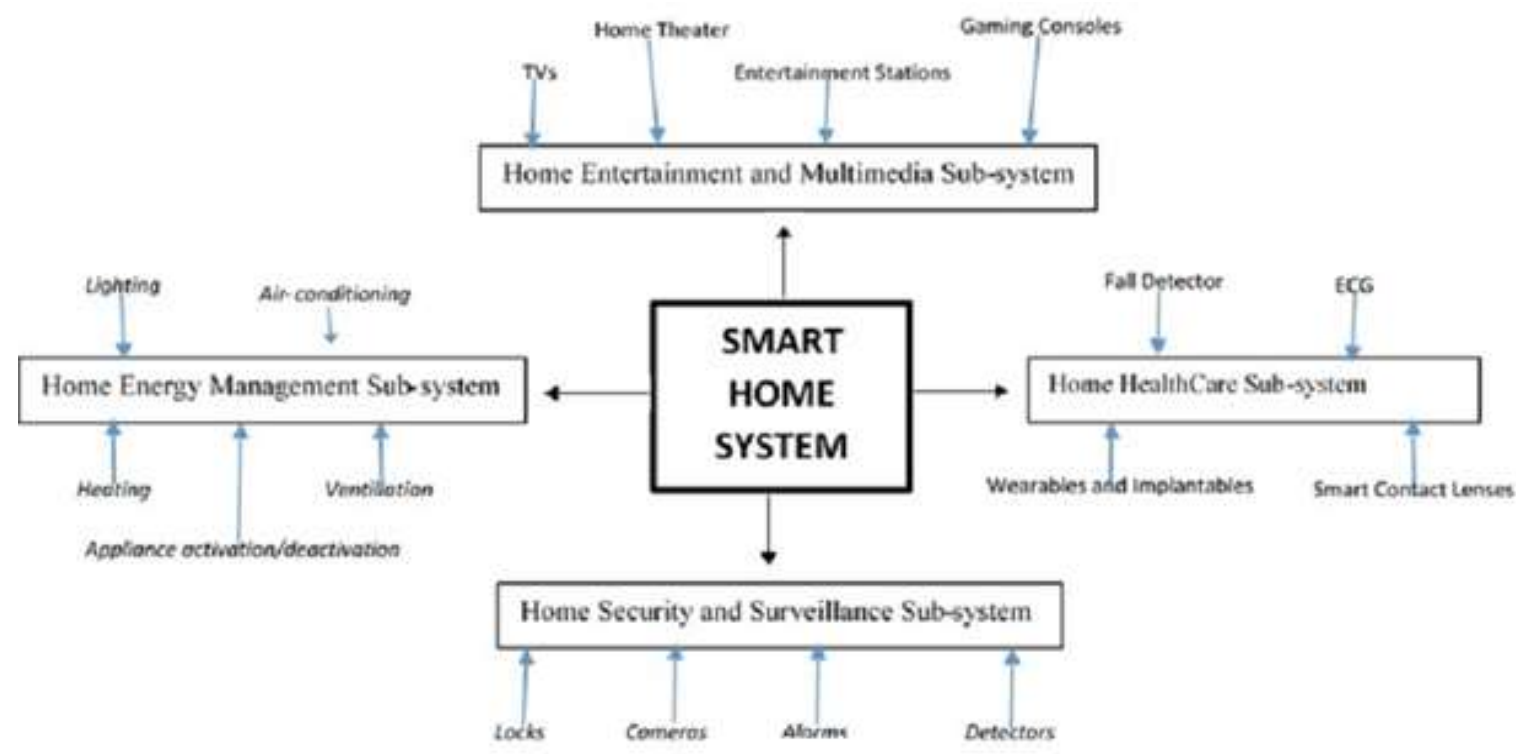

Fig 2: Smart Home Functional Parts

\subsubsection{Home Security and Surveillance Sub-system}

The implementation of security and surveillance in Smart Homes also comes with its exciting prospects as well as challenges. Such a system must be configured so as to always collect meaningful and accurate data in a reliable fashion. Such a system could also be bandwidth intensive and delay sensitive, especially when audio and video signals are involved. Wrong measurements or false event detections could occur in real-time surveillance applications when bandwidth and delay requirement are not properly addressed. The main risks associated with the interactions of entities in a Smart Home and possible countermeasures are detailed by Komninos, Philippou and Pitsillides (2014). Tongtong, Jian and Xiaochen (2012) proposed a Home Area Network (HAN) architecture with a secure access Gateway, ensuring a convenient and secure remote monitoring and control of the Smart Home. Such security measure are essential, especially when integrating loT into the Smart Home device, allowing remote access and further integration into a Smart City network. Other innovations in this area include the use of handwriting technology and recurrent Neural Network for the management of home access (Xiaohui, Guang, Bing \& Xiaobing, 2014).

\subsubsection{Home Energy Management Sub-system}

One of the main aims of the Home Energy Management Systems is to increases the energy efficiency of the smart building by controlling activation/deactivation of home appliances, lighting, heating, ventilation and air conditioning (HVAC). They employ the use of sensor networks for receiving and processing information on Smart Home power consumption patterns, and ensures that power is optimally utilized. Such systems could also be administered remotely. It is estimated that households consume a considerable part of a nations power generation (Nilsson et al, 2014). A considerable savings from electricity usage could be achieved in a Smart Home by proper energy usage understanding and management (Fabi, Spigliantini \& Corgnati, 2017). The Home Energy Management Sub-system could also help integrate solar and power storage resources to the Smart Home architecture (Al-Ali, El-Hag, Bahadiri, Harbaji \& El Haj, 2011). 


\subsubsection{Home Entertainment and Multimedia Sub-system}

According to Vanus et al (2016) and Okung-Dike, Dimitra \& David (2012), the main aim of the home entertainment and multimedia sub-system is to ensure the clean generation or creation of media contents on-the-fly and also ensure the smooth transmission of contents over multiple networks and media environments. Depending on the compression used, data rates of about $128 \mathrm{~kb} / \mathrm{s}$ to about $1 \mathrm{Mb} / \mathrm{s}$ are required by Audio applications. Also, the advent of Ultra-High Definition (UHD) contents and online straming has placed a high threshold for interconnections for entertainment purposes. Delay sensitivity and bandwidth needs must therefore be carefully considered. In addition, high capacity networked storage and media content processing resources may also be deployed.

In designing an entertainment system for a Smart Home, the frontiers of technology could be pushed (based on set budgets). For instance, a smart mirror table with an embedded camera could be used to capture occupant's facial expressions and play music which best suits the targets mood, thereby alleviating their emotions (Yuan-Chih, Shingchern \& Dwen-Ren, 2012), or the remote sharing of digital content between indoor and outdoor devices (Ti-Hsinn \& Shou-Chih, 2013).

\subsubsection{Home HealthCare Sub-system}

The main aim of the Home Health Care sub-system in a Smart Home is to monitor the occupant's physical and mental state of health, thereby ensuring a healthier and safer environment for its occupants. Devices such as blood pressure monitors, pulse meter, spirometer, PPG, oximeters, etc can be embedded into the Smart Home architecture to provide basic and advanced healthcare monitoring (Marianthi, Nikolaos \& Dimitris, 2014). A smart Home could also be designed to estimate the health and wellbeing of its inhabitant by monitoring the usage of household appliances (Suryadevara \& Mukhopadhyay, 2012) and wearables could be integrated into the Smart Home design to detect falls and other adverse incidents in the home (Brulin, Benezeth \& Courtial, 2012) and (Wang et al, 2014).

By keeping track of atmospheric readings (like CO2) in a patient's room for example, a patients habits could be mapped including eating patterns and when they take a walk, without invading their private space. Changes to such observed patterns could be spotted and action taken remotely. The HealthCare sub-system could even be used to help occupants in mundane home activities such as cooking (Camier, Giroux, Bouchard \& Bouzouane, 2013). Due to the increasingly aging population, the need and use of such systems in Smart Homes is projected to increase steadily. It is imperative that adequate security and safety measures are carefully taken due to the sensitive nature of this sub-system (Marianthi et al, 2014). Controls, data integrity and quality must strictly meet pre-set medical and technical standards. Authentication and authorization is especially important in an loT-based Healthcare sub-system (Moosavi et al, 2015)

Specific communications protocols are best suited for specific implementation scenarios within the Smart Home subsystems. The use of similar communications requirements across the entire Smart Home architecture is both uneconomical and froth with several major demerits (Zhen-ya, 2014). 
The Table below gives a summary of the different sub-systems, their characteristics/requirements and what communication protocols best suites their implementation (Mendes et al, 2015);

\begin{tabular}{|c|c|c|c|c|c|c|}
\hline & $\begin{array}{l}\text { Smart Home } \\
\text { Functional } \\
\text { Area }\end{array}$ & $\begin{array}{l}\text { Data Rate } \\
\text { Requirements }\end{array}$ & $\begin{array}{l}\text { Bandwidth } \\
\text { Requirements }\end{array}$ & $\begin{array}{l}\text { Security } \\
\text { Considerations }\end{array}$ & $\begin{array}{l}\text { Power } \\
\text { Considerations }\end{array}$ & $\begin{array}{l}\text { Application } \\
\text { Area }\end{array}$ \\
\hline 1 & $\begin{array}{l}\text { Home } \\
\text { Security and } \\
\text { Surveillance } \\
\text { Sub-system }\end{array}$ & $\begin{array}{l}200 \mathrm{Kbps} \\
54 \mathrm{Mbps}\end{array}$ & Low - High & Important & High & $\begin{array}{l}\text { Alarms, CCTV } \\
\text { cameras, HD } \\
\text { video } \\
\text { surveillance. }\end{array}$ \\
\hline 2 & $\begin{array}{l}\text { Home Energy } \\
\text { Management } \\
\text { Sub-system }\end{array}$ & $\begin{array}{l}\text { 2.1Kbps } \\
\text { 250Kbps }\end{array}$ & Low & Important & Low & $\begin{array}{l}\text { HVAC control, } \\
\text { smart meters, } \\
\text { home } \\
\text { appliance } \\
\text { activation \& } \\
\text { deactivation. }\end{array}$ \\
\hline 3 & $\begin{array}{l}\text { Home } \\
\text { Entertainmen } \\
t \quad \text { and } \\
\text { Multimedia } \\
\text { Sub-system }\end{array}$ & $\begin{array}{l}250 \mathrm{Kbps} \\
500 \mathrm{Mbps}\end{array}$ & $\begin{array}{l}\text { Medium (stereo } \\
\text { audio) } \\
\text { High (SDTV) } \\
\text { Very high (HD } \\
\text { video) }\end{array}$ & Minimal & High & $\begin{array}{l}\text { Audio, SDTV, } \\
\text { HD Video. }\end{array}$ \\
\hline 4 & $\begin{array}{l}\text { Home } \\
\text { HealthCare } \\
\text { Sub-system }\end{array}$ & 12bps - 15Mbps & Low - High & Very Important & Very High & $\begin{array}{l}\text { Pulse } \\
\text { Oximeter, } \\
\text { EMG, wrist } \\
\text { straps, fall } \\
\text { detectors. }\end{array}$ \\
\hline
\end{tabular}

\section{DATA PRESENTATION}

While it is true that the adoption of the Smart Home concept comes with numerous advantages including major savings in terms of energy consumption, innovative healthcare applications, better home security, etc, a careful evaluation of the smart home architectural design, shows that the design and deployment of Smart Home system is faced with major challenges (see FIG. 3). These include:

\subsection{Slow adoption of the Smart Home concept}

While it is projected that the industry supporting Smart Homes is worth billions of dollars and expected to increase tremendously, yet a wide-spread adoption of the Smart Home concept is yet to take place (Smireka, Zimmermanna \& Beigl, 2016). This may be traced to high cost of ownership, poor manageability, and rigidity of interconnected devices, platform fragmentation amongst others.

\subsection{Information Security and Data Ownership Risks}

The Smart Home generate a lot of information and the issue of data ownership risk remains a contending issue. Many of such generated information is sent to equipment vendors. How can one ensure that such information is not misused or misapplied? In other to maximize the advantages of the ever increasing amounts of data, there is an urgent need to 
propose new workable methods and techniques for effective data management and analysis, geared towards generating information that can assist in managing the utilization of resources intelligently and dynamically (Gaur, Scotney, Parr \& McClean, 2015). In addition, there is a major security risk with Smart Home remote capabilities and its integration to the Internet. Furthermore, relevant software patches hardly ever reach users with older or low-budget devices, making the entire Smart Home system vulnerable to attacks.

\subsection{High Cost of technology deployment and maintenance}

There is also the issue of high cost of technology deployment and maintenance (Fabi et al, 2017). Smart Homes generally consist of sensor and actuator networks. Complicated procedures are required for calibrating, configuring and maintaining these sensitive devices (Sun et al, 2013). In addition, Users do not wish to get locked into one specific vendor. They would rather not use proprietary hardware/software devices whose protocol use may fade or that present future difficulties in customization or interconnection. Furthermore, collating raw sensor data from Smart Home environment can be complex and irregular to encode into predefined scenarios and deviant behaviour is also difficult to identify due to varried factors. These issues could present major problems in formulating equations for Smart Home system operations (Ghayvat et al, 2015).

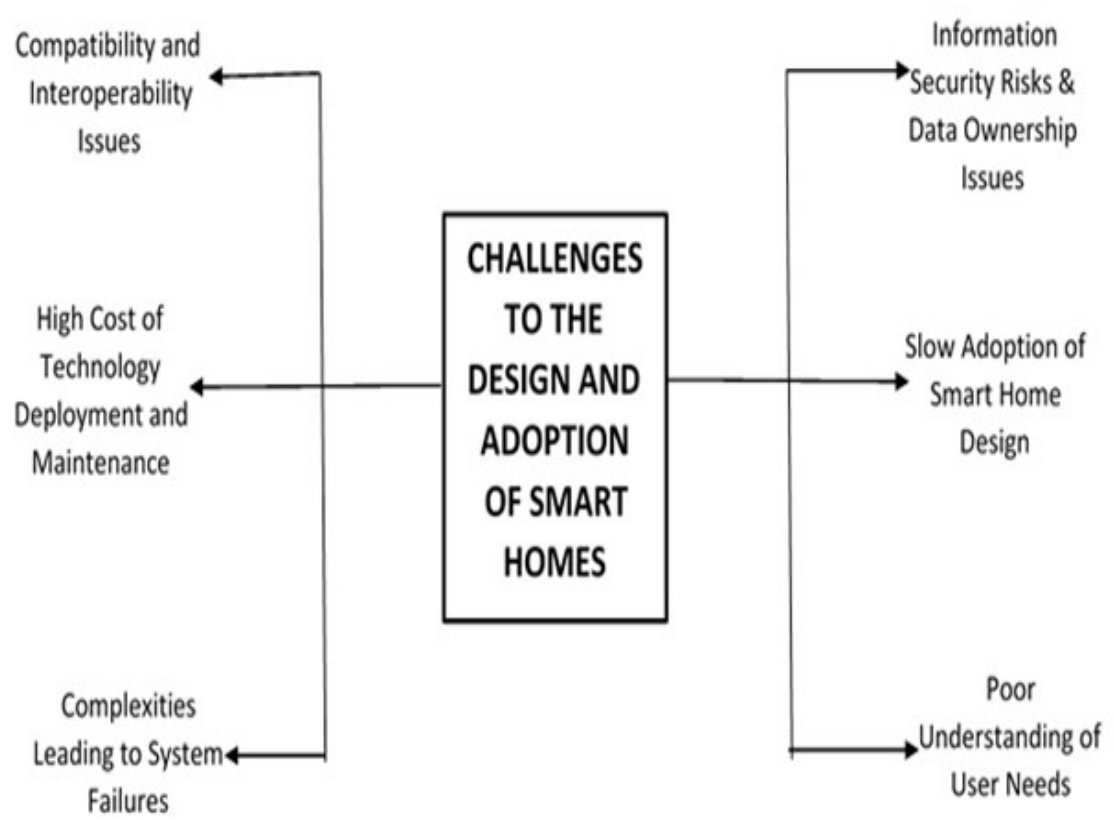

Fig 3: Challenges to Design and Adoption of Smart Home

\subsection{Poor Understanding of User Needs}

The Smart Home architecture is often beset by complex user interfaces (Fabi, 2017) and a poor understanding of evolving user needs. Users need to feel in control of their home environment. Complex or difficult user interfaces could easily put users off the technology. In the same vein, user needs are ever evolving and a good understanding of this evolution pattern will aid in building more acceptable Smart Homes (Leea \& Trimi, 2016). 


\subsection{Compatibility and Interoperability Issues}

Low interoperability between different smart home systems and the Challenges associated with installing and integrating solutions into already existing homes remains a major challenge (Smireka et al, 2016). Interoperability is a vital requirement for data communication (Gao et al, 2012). A major challenge with Smart Home design is the issue of platform fragmentation and a deficiency in generally accepted technical standard. Although several standards organizations (IETF, OASIS, etc) have focused research to provide interoperable protocols, yet they do not address all technology requirements to create and adapt end-user applications without understanding the underlying technological details. Brush et al (2011) asserts that device interoperability standards, while allowing users to purchase devices from diverse vendors, yet are an insufficient measure alone since they provide no basis for coordinating across device platforms. It is also pertinent to note that centralized schemes are generally not scalable, this implies that a failure of an agent device could result in a dysfunctional Smart Home system (Sun et al, 2013). Indeed real interoperability requires the harmonization of multiple protocol standards.

\subsection{Complexities Leading to System Failures}

Smart Homes consist of large number of distributed devices, which are networked and integrated into the Smart Home system. This could typically result in a complicated system and different components may have different objectives that are competing. This could lead to system failures if not handled properly. Furthermore, Magruk (2015) described the phenomenon of uncertainty in the face of technological megatrends and unique challenges associated with them. The Internet of Things (IoT) is a big, complex, dynamic system with specific characteristics, dimensions, structures and behaviours. Integrating the loT into the Smart Home architecture also introduces uncertainty levels to the overall architecture. It is imperative to note however that no research procedures or mathematical calculations could totally eliminate uncertainty.

\section{DISCUSSION OF FINDINGS}

Due to above enumerated challenges besetting the Smart Home architecture, a universal framework is needed in other to amongst other things address the integration of different backend and platforms. The following are proposed (see FIG. 4):

\subsection{Integrating Innovative Technologies into Smart Home Architecture}

In other to reduce the computational demands on the Smart Home system, central specialized computers situated outside the home could be used to process relevant information from the Smart Homes. Command signals are thereafter sent to components in the Smart Homes as required. However, in other to mitigate system failures, the Smart Home components could be configured so that commands could be sent directly to actuators in the event of a network failure. In addition to cloud computing, artificial neural network technology could be embedded into the Smart Home design to further enhance and increase its capabilities. This approach is especially useful when the loT is fully integrated into the Smart Home and such Smart Homes are further knitted together to develop a smart city infrastructure. It must however be noted that such Smart Home systems which integrate neural networks require centralized implementation schemes, process very large amounts of information and therefore not presently advisable for distributed, scalable and robust Smart Home designs (Sun et al, 2013).

\subsection{Product Compatibility and Platform Interoperability}

It is imperative that vendor products in the Smart Home field are compatible (both forward and backward compatibility) with each other and remain that way in the foreseeable future. Policymakers could play a pivotal role in this regards. Furthermore, in other to overcome the interoperability issues between different protocols/applications, a universal multipurpose gateway can be deployed as a communications protocol translator, since they support multiple protocols (Fernandes, Jung \& Prakash, 2016). The gateway can also be used to connect the Smart Home to the Internet and the outside world. 
Further, a software tool could be used to mesh up various devices and Internet services, thereby creating new applications and extensive flexibility in service oriented device-centric environments. This ensures a lightweight solution which users with little technical knowledge can use easily. For instance, the Niagara Framework is a Java-based framework that allows the design of Smart Homes using different devices/systems from different vendors. Here, every device component is treated as an object and ensures that every object can run from a standard browser regardless of hardware/operating system platforms or protocol/communication standard. The Niagara Framework could therefore be used to reap all the benefits associated with the full integration of loT in the Smart Home.

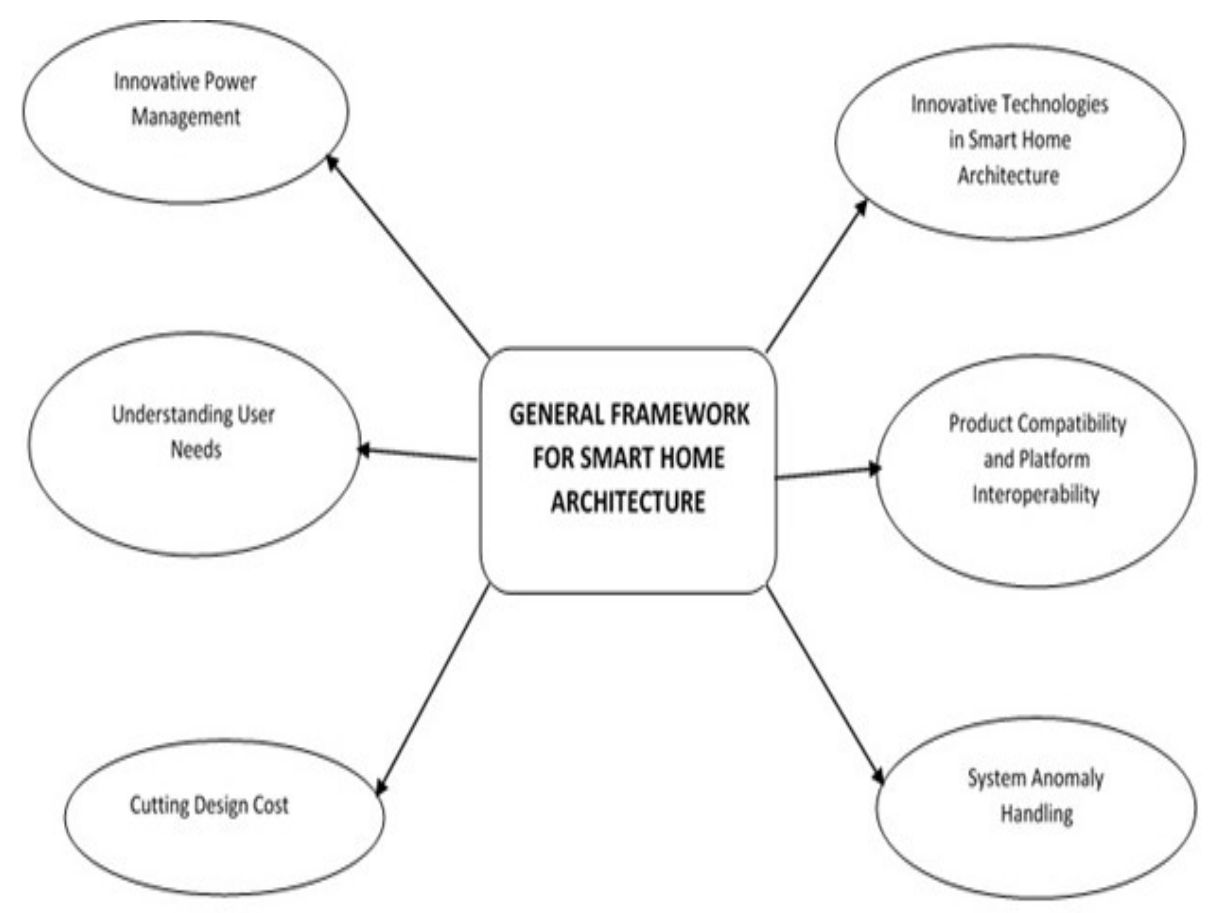

Fig. 4: Proposed Framework for Mitigating Challenges to Smart Home Architecture

\subsection{Innovative Power Management In Smart Home Architecture}

Smart Home Energy Management Schemes (SHEMS) could be used to manage energy consumption in Smart Homes. They can be used to amongst other things save power when rates are low, re-inject saved power into the Home system when power rates are higher or switch to alternate power sources such as solar power, etc (Vanus et al, 2016). A methodology aimed at improving Smart Home Information management through improved device interoperability and network efficiency can also be adopted (Margherita et al, 2013). In addition, a framework for managing the power consumption of appliances in Smart Homes within the same neighborhood as proposed by Roberto et al (2014) can be put to good use. Furthermore, the use of self-powered sensors, transmitters and actuators could be employed. These devices make use of energy converters such as piezo-generators, solar cells, electromagnetics, thermocuples, etc, which generate their power requirements from harnessing energy from its operational environment like motion, temperature and light differences. The deployment of such technology in the design of the Smart Home will help prolong the working life of such sensors and further reduce frequency of maintenance and associated cost. 


\subsection{Understanding User Needs}

In addition, a major gap in today's Smart Home design lies in understanding the ever evolving consumers' behavior, accurately documenting and harnessing these behaviors and correctly using them in the Smart Home architecture. The deployment of technologies such as Universal Remote Console (URC) and the Universal Control Hub (UCH) could be successfully used to ensure that users feel comfortable and in control of home control systems (Smireka et al, 2016). In addition, the Eclipse Smart Home (ESH) offers a flexible and modularized structure with special focus on heterogeneous environments, wherein the principles of modularization and hot deployment are employed in loading new components (Smireka et al, 2016). The URC concepts can also be integrated into the ESH approach. Hand gesture interface systems could also be integrated into the Smart Home design to further simplify home controls and make them fun, while adding greater capabilities.

\subsection{Cutting Smart Home Design Cost}

In other to tackle the high cost of deployment and further encourage widespread adoption of Smart Home technologies, innovative yet effective new ways of applying technology in the Smart Home architecture could be explored. For instance, the use of image processing based mechanism, generic email service protocols and providers to provide remote access, security and surveillance for the Smart Home (Pooshkar, Rohit \& Mahesh, 2016). This ingenious mechanism for access control and surveillance is cost effective, efficient, reliable, robust and can be easily deployed on generic hardware.

Adequate and systematic planning of sensor placement in the Smart Home can drastically reduce the number of needed sensors for activity recognition, thereby cutting complexity and costs associated with the deployment and maintenance of the sensor network. Choosing the right communication protocol for the Smart Home sensor network is also paramount in ensuring a durable and dependable design. Information gain can be used to reduce the number of sensors needed for activity recognition and also guide future sensor installation for specific activities in future Smart Homes.

\subsection{Smart Home System Anomaly Handling}

In a typical Smart Home heterogeneous environment, large variety of anomalies could occur in a Smart Home system (such as hardware, software, network, operator or context faults), possibly disrupting the functions of critical and essential applications (Pardo, Espes \& Le-Parc, 2016). It is therefore imperative to integrate an anomaly management system into the overall architecture design of a smart home system, thereby making it quite robust and increase its interoperability capacity and dependability. 


\section{CONCLUDING REMARKS}

This paper considered the general architecture of Smart Homes, its functional parts and sub-systems. It also outlined major requirements needed in implementing various sub-systems in the Smart Home. Furthermore, a close look at present challenges to Smart Home design were considered and a possible framework was proposed to help overcome identified challenges in the design of the Smart Home architecture. It has been established that while designing a Smart Home architecture, careful considerations must be made in choosing device platforms and appropriate communication protocols, including user requirements, reliability, compatibility/support, ease of integration/interoperability, design costs and long-term investment issues. If these considerations are carefully factored into the Smart Home architecture, it will help overcome the challenges to Smart Home design and adoption.

\section{CONTRIBUTIONS TO KNOWLEDGE}

Smart Homes will grow ever more intelligent, with mind boggling capabilities, especially when fully integrated with the Internet of Things (loT) and Artificial Intelligence, and locked into a Smart City-Wide Network. Such a scenario will present us with endless opportunities and vastly new areas of interest. In light of the literature review and study evidence, the following recommendations are suggested:

1. There is the urgent need for the union of Wireless Sensor Network (WSN), the Internet and distributed computing for greater research advancement.

2. A concerted effort on the part of governmental agencies is needed to force/cajole/prod vendor/players in the smart home industry to harmonise platforms and protocols in other to ensure backward/forward compatibility and interoperability.

3. It is expected that smart homes will make a radical change to the way people manage their private lives, interact with one another and their environment in the future. It is therefore imperative that governments build and adopt a roadmap towards harnessing all the benefits of smart homes towards greater nation building. 


\section{REFERENCES}

1. Al-Ali A. R., El-Hag A., Bahadiri M., Harbaji M. \& El Haj A. Y. (2011) "Smart Home Renewable Energy Management System". Energy Procedia, Volume 12, 2011, Pages 120-126. DOl: http://dx.doi.org/10.1016/i.egypro.2011.10.017

2. Brulin D., Benezeth Y. \& Courtial E. (2012) "Posture Recognition Based on Fuzzy Logic for Home Monitoring of the Elderly". IEEE Trans. Inf. Technol. Biomed. 2012, 16, 974-982.

3. Camier R. T., Giroux S., Bouchard B. \& Bouzouane A. (2013) "Designing a NIALM in Smart Homes for Cognitive Assistance". Procedia Computer Science, Volume 19, 2013, Pages 524-532. DOI: http://dx.doi.org/10.1016/j.procs.2013.06.070

4. De Silva L.C.; Morikawa C. \& Petra I.M. (2012) "State of the art of smart homes". Engineering Applications of Artificial Intelligence, Volume 25, Issue 7, October 2012, Pages 1313-1321. DOI: http://doi.org/10.1016/j.engappai.2012.05.002.

5. Eugeny I. B. (2015) "The Distinctive Features of "Smart" Buildings". Procedia Engineering, Volume 111, 2015, Pages 103-107. DOI: http://dx.doi.org/10.1016/j.proeng.2015.07.061

6. Fabi V., Spigliantini G. \& Corgnati P. S. (2017) "Insights on Smart Home concept and occupants' interaction with building controls". 8th International Conference on Sustainability in Energy and Buildings, SEB-16, 11-13 September. 2016, Turin, ITALY. Energy Procedia Volume 111, March 2017, Pages 759-769. DOI: http://doi.org/10.1016/j.egypro.2017.03.238.

7. Gaur A., Scotney B., Parr G. \& McClean S. (2015) "Smart City Architecture and its Applications Based on loT". Procedia Computer Science, Volume 52, 2015, Pages 1089-1094. DOl: http://dx.doi.org/10.1016/j.procs.2015.05.122

8. Ghayvat H., Mukhopadhyay S., Gui X. \& Suryadevara N. (2015) "WSN- and IOT-Based Smart Homes and Their Extension to Smart Buildings". Sensors 2015, Volume 15, 10350-10379 Dol:10.3390/s150510350.

9. Jucevičius R.; Patašienè I. \& Patašius M. (2014) "Digital Dimension of Smart City: Critical Analysis". Procedia Social and Behavioral Sciences, Volume 156, 26 November 2014, Pages 146-150. DOI: http://dx.doi.org/10.1016/i.sbspro.2014.11.137

10. Komninos N., Philippou E. \& Pitsillides A. (2014) "Survey in Smart Grid and Smart Home Security: Issues, Challenges and Countermeasures". IEEE Communications Surveys \& Tutorials 2014. Volume: 16, Issue: 4. Dol: 10.1109/COMST.2014.2320093.

11. Leea S. M. \& Trimi S. (2016) "Innovation for creating a smart future". Journal of Innovation \& Knowledge (in press). DOI: http://dx.doi.org/10.1016/j.jik.2016.11.001

12. Magruk A. (2015) "The Most Important Aspects of Uncertainty in the Internet of Things Field - Context of Smart Buildings". Procedia Engineering, Volume 122, 2015, Pages 220-227. DOl: http://dx.doi.org/10.1016/.jproeng.2015.10.028

13. Margherita P., Michele G., Alessandra P. \& Andrea C. (2013) "Smart Home Information Management System for Energy-Efficient Networks". 14th Working Conference on Virtual Enterprises, (PROVE), Sep 2013, Dresden, Germany. Springer, IFIP Advances in Information and Communication Technology, AICT- Volume 408, pp.393401, 2013, Collaborative Systems for Reindustrialization. Dol: 10.1007/978-3-642-40543-3_42

14. Marianthi T., Nikolaos T. \& Dimitris G. (2014) "Smart Home Solutions: Privacy Issues". In Handbook of Smart Homes, Health Care and Well-Being. Page: 67-81. Springer: Cham, Switzerland, 2014. DOI: 10.1007/978-3-31901583-5_5 
15. Mendes D. P. T., Godina R., Rodrigues M. G. E., Matias C. O. J. \& Catalão P. S. J. (2015) "Smart Home Communication Technologies and Applications: Wireless Protocol Assessment for Home Area Network Resources". Energies 2015, 8, 7279-7311. DOI:10.3390/en8077279.

16. Moosavi R. S., Gia N. T., Rahmani A., Nigussie E., Virtanen S., Isoaho J, Tenhunen H. (2015) "SEA: A Secure and Efficient Authentication and Authorization Architecture for loT-Based Healthcare Using Smart Gateways". Procedia Computer Science, Volume 52, 2015, Pages 452-459. DOI: http://dx.doi.org/10.1016/j.procs.2015.05.013

17. Nilsson A, Bergstad C.J., Thuvander L., Andersson D., Andersson K. and Meiling P. (2014) "Effects of continuous feedback on households' electricity consumption: Potentials and barriers". Applied Energy, Volume 122, 1 June 2014, Pages 17-23. DOI: http://doi.org/10.1016/j.apenergy.2014.01.060

18. Okung-Dike N., Dimitra S. \& David K. H. (2012) "Cloud-based architecture for deploying ultra-high-definition media over intelligent optical networks". In Proceedings of the 2012 16th International Conference on Optical Network Design and Modeling (ONDM), Colchester, UK, 17-20 April 2012. DOI: 10.1109/ONDM.2012.6210201.

19. Pooshkar R., Rohit R. \& Mahesh C. (2016) "Email based remote access and surveillance system for smart home infrastructure". Perspectives in Science, Volume 8, September 2016, Pages 459-461. DOl: http://dx.doi.org/10.1016/j.pisc.2016.04.104

20. Pardo E., Espes D. \& Le-Parc P. (2016) "A Framework for Anomaly Diagnosis in Smart Homes Based on Ontology". Procedia Computer Science, Volume 83, 2016, Pages 545-552. DOl: http://dx.doi.org/10.1016/j.procs.2016.04.255

21. Praveen V. \& Bagavathi S. P. (2016) "Design of IoT Systems and Analytics in the Context of Smart City Initiatives in India". Procedia Computer Science, Volume 92, 2016, Pages 583-588. DOl: http://dx.doi.org/10.1016/j.procs.2016.07.386

22. Roberto C., Giuseppe C., Pilloni \& Luigi A. (2014) "Energy consumption management in Smart Homes: An M-Bus communication system". In Proceedings of the 2014 International Conference on Telecommunications and Multimedia (TEMU), Heraklion, Greece, 28-30 July 2014. DOI: 10.1109/TEMU.2014.6917740.

23. Smireka L., Zimmermanna G. \& Beigl M. (2016) "Just a Smart Home or Your Smart Home - A Framework for Personalized User Interfaces Based on Eclipse Smart Home and Universal Remote Console". Procedia Computer Science, Volume 98, 2016, Pages 107-116. DOI: http://dx.doi.org/10.1016/j.procs.2016.09.018

24. Soyinka O., Siua M. W. K, Lawansonb T. \& Adeniji O. (2016) "Assessing smart infrastructure for sustainable urban development in the Lagos metropolis". Journal of Urban Management, Volume 5, Issue 2, December 2016, Pages 52-64. DOI: http://dx.doi.org/10.1016/j.jum.2017.01.001

25. Sun Q., Yu W., Kochurov N., Hao Q. \& Hu F. (2013) "A Multi-Agent-Based Intelligent Sensor and Actuator Network Design for Smart House and Home Automation". Journal of Sensor and Actuator Networks, 2013, Volume 2, Pages: 557-588. Dol: 10.3390/jsan2030557

26. Suryadevara, N. \& Mukhopadhyay S. (2012) "Wireless Sensor Network Based Home Monitoring System for Wellness Determination of Elderly". IEEE Sens. J. 2012, 12, 1965-1972

27. Ti-Hsinn Y. \& Shou-Chih L. (2013) "A Remote Control and Media Sharing System Based on DLNA/UPnP Technology for Smart Home". In: Park J., Ng JY., Jeong HY., Waluyo B. (eds) Multimedia and Ubiquitous Engineering. Lecture Notes in Electrical Engineering, vol 240. pp. 329-335. Springer, Dordrecht. DOl: 10.1007/978-94-007-6738-6_41.

28. Tongtong L., Jian R. \& Xiaochen T. (2012) "Secure Wireless Monitoring and Control Systems for Smart Grid and Smart Home". IEEE Wireless Communications, Volume: 19, Issue: 3, June 2012, Page: 66-73. DOI: 10.1109/MWC.2012.6231161. 
29. Vanus J., Vojcinak P., Martinek R., Kelnar M., Machacek Z., Bilik P., Koziorek J., Zidek J. (2016) "Building heating technology in Smart Home using PI System management tools". Perspectives in Science, Volume 7, March 2016, Pages 114-121. DOl: http://dx.doi.org/10.1016/j.pisc.2015.11.019

30. Wang J., Zhang Z., Li B., Lee S. \& Sherratt R. (2014) "An enhanced fall detection system for elderly person monitoring using consumer home networks". IEEE Trans. Consum. Electron. 2014, 60, $23-29$.

31. Yuan-Chih Y., Shingchern D. Y. \& Dwen-Ren T. (2012) "Magic mirror table for social-emotion alleviation in the smart home". IEEE Transactions on Consumer Electronics 2012, Volume: 58, Issue: 1, Pages: 126-131. DOI: 10.1109/TCE. 2012.6170064.

32. Zhang D.; Shah N. \& Papageorgiou L.G. (2013) "Efficient energy consumption and operation management in a smart building with microgrid". Energy Conversion and Management, Volume 74, October 2013, Pages 209-222. DOl: http://doi.org/10.1016/j.enconman.2013.04.038.

33. Zhen-ya L. (2014) "Hardware Design of Smart Home System based on ZigBee Wireless Sensor Network". AASRI Procedia, Volume 8, 2014, Pages 75-81. DOI: http://dx.doi.org/10.1016/j.aasri.2014.08.013 